\title{
Ectrodactyly-Ectodermal Dysplasia-Cleft Syndrome
}

National Cancer Institute

\section{Source}

National Cancer Institute. Ectrodactyly-Ectodermal Dysplasia-Cleft Syndrome. NCI

Thesaurus. Code C148261.

A rare form of ectodermal dysplasia, inherited in an autosomal dominant fashion,

manifesting with varying degrees of severity, ectrodactyly and cleft lip/palate. 